\title{
La luxation columno-spatulaire, une luxation tarsométatarsienne rare
}

\author{
Medial and lateral metatarsal dislocation, a rare tarsometatarsal dislocation
}

\author{
H. Trouvain · J.-B. Lézy $\cdot$ A. K. Djobokou
}

Reçu le 5 avril 2018; accepté le 19 août 2018

(C) SFMU et Lavoisier SAS 2018
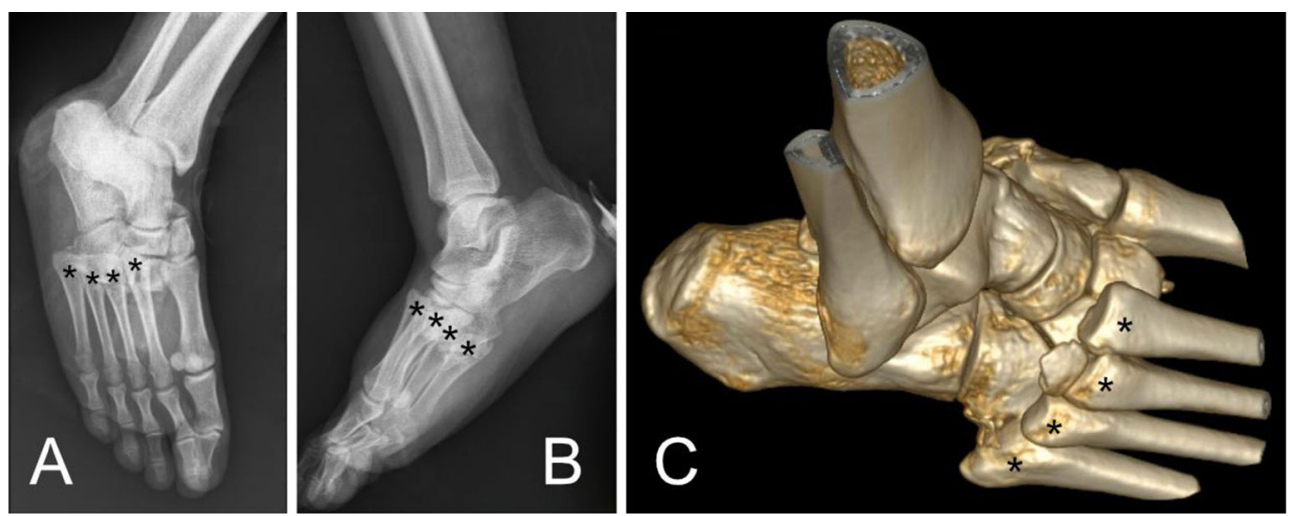

Fig. 1 Radiographie du pied de face non stricte (A) et de profil (B) : luxation des têtes des 4 derniers métatarsiens (étoiles) ; C. Reconstitution en 3 dimensions à partir du scanner du pied

Un homme de vingt-deux ans est victime d'un accident de la voie publique de haute cinétique. Le patient se plaint d'une douleur élective du pied droit avec une déformation du dos du pied sans effraction cutanée. La radiographie retrouve une luxation tarsométatarsienne avec une fracture complexe de l'os naviculaire et une probable fracture des os cunéiformes et des têtes des métatarsiens. Le scanner complémentaire confirme une luxation de type columno-spatulaire dorso-externe des cinq métatarsiens (classification de Quénu) associée à une fracture pluri-fragmentaire déplacée de l'os naviculaire, une fracture de l'os cuboïde ainsi qu'une fracture-arrachement des deux premiers cunéiformes (Fig. 1). Le reste du bilan lésionnel est négatif. La prise en charge sera chirurgicale : réduction et ostéosynthèse per-cutanée par brochage multiple puis immobilisation par botte plâtrée pendant six semaines. Les suites ont été simples.

La luxation tarsométatarsienne ou du Lisfranc est une pathologie rare $(1 / 55000)$, survenant essentiellement lors d'un accident de la voie publique (pied sur la pédale de

\footnotetext{
H. Trouvain $(\bowtie) \cdot$ J.-B. Lézy $\cdot$ A. K. Djobokou

Service d'accueil des urgences, SMUR

Centre hospitalier d'Armentières,

112, rue Sadi-Carnot, F-59280 Armentières, France

e-mail : h.trouvain@ch-armentieres.fr
}

frein). La luxation columno-spatulaire est un type de luxation tarsométatarsienne intéressant à la fois la colonne (premiers cunéiforme et métatarse) et la spatule (quatre autres métatarses) [1]. Ce type de luxation est souvent complexe car le plus souvent associé à des fractures du tarse et des métatarses. C'est une pathologie sous-diagnostiquée (20\%) par manque de puissance de la radiographie. Toute suspicion radiologique de luxation tarsométatarsienne doit être confirmée par un scanner complémentaire. En outre celui-ci permet de déceler des lésions associées et d'adapter la prise en charge thérapeutique. Le traitement est chirurgical : réduction et ostéosynthèse puis immobilisation par botte plâtrée. Ce diagnostic est important à établir car le pronostic fonctionnel est très défavorable pour les cas négligés ou mal traités (douleurs chroniques, cals vicieux, raideurs articulaires) [2].

\section{Références}

1. Quenu E, Kuss G (1909) Étude sur les luxations du métatarse. Rev Chir 39:281-6

2. Mulier T, De Haan J, Vriesendorp P, Reynders P (2010) The treatment of Lisfranc injuries: review of current literature. Eur $\mathrm{J}$ Trauma Emerg Surg 36:206-16 